\title{
Sperm recovery and IVF outcome after surgical sperm retrieval in azoospermia: our experience
}

\author{
Seema Rai ${ }^{1}$, P. S. Rao ${ }^{2 *}$, Prajwal S. ${ }^{2}$
}

\author{
${ }^{1}$ Department of Obstetrics and Gynecology, Army Hospital R. R., New Delhi, India \\ ${ }^{2}$ Department of Obstetrics and Gynaecology, Command Hospital A. F., Bangalore, Karnataka, India
}

Received: 25 December 2017

Accepted: 30 December 2017

\section{*Correspondence:}

Dr. P. S. Rao,

E-mail: doctorpsrao@gmail.com

Copyright: (c) the author(s), publisher and licensee Medip Academy. This is an open-access article distributed under the terms of the Creative Commons Attribution Non-Commercial License, which permits unrestricted non-commercial use, distribution, and reproduction in any medium, provided the original work is properly cited.

\section{ABSTRACT}

Background: In the last decade, male factor infertility has been recognized as a significant global problem, which is on an increase. Till date, very few options are available for ameliorating male factor infertility. Development of intracytoplasmic sperm injection has revolutionized the treatment of males with very low sperm counts or with poor quality sperm. However, patients with a diagnosis of azoospermia had no option but to resort to adoption or use of donor sperm. The clinical management of men with azoospermia seeking fertility has been a challenge for andrologists, urologists, and reproductive medicine specialists alike. The older approaches denied such couple the possibility of having own genetic children. Surgical sperm retrieval and intracytoplasmic sperm injection have successfully transformed the treatment of male infertility so that now most of the men can father their own children. The aim of this study is to present the outcome of IVF with surgically retrieved sperm from azoospermic men.

Methods: This study was a retrospective analysis of 100 cycles using surgically retrieved sperm. Outcome measures were fertilization rate (FR) and implantation rate (IR) per transfer.

Results: Sperm retrieval rate in our study was $76 \%$ when Per cutaneous epididymal sperm aspiration (PESA) and Testicular sperm extraction (TESE) are combined. Our data demonstrated similar outcome between the use of epididymal or testicular sperm in men with azoospermic. There was no statistical difference concerning fertilization rate, embryo quality and pregnancy rate between ICSI with surgically retrieved sperm and ejaculated sperms.

Conclusions: Surgical sperm retrieval is a feasible and successful procedure. Spermatozoa can be retrieved from the testis in up to $76 \%$ of patients, even in cases with severe disorders of spermatogenesis and deranged hormonal profile. Comprehensive genetic counseling is important for infertile couples deciding for surgical sperm retrieval, since genetic disorders, as yet undetected, may be passed on to the following generation with multiplying variety.

Keywords: Azoospermia, Cryptozoospermia, Percutaneous epididymal sperm aspiration, Surgical sperm retrieval, Testicular sperm extraction

\section{INTRODUCTION}

Birth of Louise Brown in 1978 was a breakthrough in the field of reproductive medicine. Since then numerous tests and techniques have been developed to improve the treatment of infertility. However, all these developments were focused towards female factor infertility, and male infertility was sadly neglected. In the last decade, male factor infertility has been recognized as a significant global problem, which is on an increase. Till date, very few options are available for ameliorating male factor infertility. Development of intracytoplasmic sperm injection has revolutionized the treatment of males with very low sperm counts or with poor quality sperm. 
However, patients with a diagnosis of azoospermia had no option but to resort to adoption or use of donor sperm. The clinical management of men with azoospermia seeking fertility has been a challenge for andrologists, urologists, and reproductive medicine specialists alike. The older approaches denied such couple the possibility of having own genetic children. Surgical sperm retrieval and intracytoplasmic sperm injection have successfully transformed the treatment of male infertility so that now most of the men can father their own children.

Azoospermia is the complete absence of sperm in the sediment of a centrifuged $(3000 \mathrm{~g}$ for $15 \mathrm{~min}$ at room temperature) ejaculated semen sample examined on two different occasions by phase contrast optics at $\times 200$ magnification with appropriate sexual abstinence. ${ }^{1}$ Cryptozoospermia is defined by the presence of very small number of live sperm in pellet and distinguishes it from azoospermia. ${ }^{2}$ The finding of live sperm may allow ICSI to be performed with ejaculated sperm, thus obviating the need of surgical sperm retrieval. Sperms usable for ICSI can be found in up to $10 \%$ of patients with an initially azoospermic semen specimen after an extended analysis of the centrifuged specimen. ${ }^{3}$

It is also important to rule out retrograde ejaculation by examining a post-ejaculate urine specimen. ${ }^{4}$ The diagnosis of retrograde ejaculation is confirmed by presence of any sperm in the urine, post-ejaculation in men with azoospermia. Use of an alpha agonist (pseudoephedrine or other alpha agonist) before ejaculation may be helpful in cases of retrograde ejaculation. These drugs close the bladder neck during orgasm and convert retrograde into ante-grade ejaculation. This entity is more commonly seen in diabetic men. ${ }^{5}$

The incidence of azoospermia without implying an underlying etiology is $1 \%$ amongst men and close to $20 \%$ in men seeking fertility treatment. ${ }^{2,4}$

Azoospermia is classified as obstructive (reproductive tract obstruction) or non-obstructive (inadequate production of spermatozoa by testis). Mechanical blockage occurring anywhere along the reproductive tract, including the vas deferens, epididymis, and ejaculatory duct can result in obstructive azoospermia. Obstructive azoospermia (OA) causes azoospermia in up to 20 to $40 \%$ of cases. In OA both reconstructive procedures and surgical retrieval are highly successful. ${ }^{6-8}$ Non-Obstructive azoospermia (NOA) is associated with a spectrum of various severe and untreatable conditions associated with an intrinsic testicular impairment. ${ }^{9}$ Parameters like history, physical examination, testicular volume and hormonal analysis (FSH, LH, TSH, Testosterone) and even the histology of a diagnostic biopsy are only indicative and not always predictive in diagnosing the cause of azoospermia because patients with maturation arrest (MA) generally have welldeveloped and normal-sized testes and normal hormonal levels. ${ }^{10,11}$ Levels of $\mathrm{FSH}>7.6 \mathrm{mIU} / \mathrm{ml}$ are found in about $89 \%$ of men with NOA but only if the levels are greater than twice the upper normal limit do they provide a reasonably precise diagnosis of testicular failure. ${ }^{12}$

Ejaculates of men with azoospermia usually present with normal volume $(>1.5 \mathrm{ml})$ and $\mathrm{pH}(>7.2)$. But as azoospermia is defined based on the absence of spermatozoa in a given ejaculate, correct and precise laboratory technique is important to reduce analytical error and enhance precision when analyzing semen specimens. ${ }^{13}$

Genetic evaluation especially karyotype and $\mathrm{Y}$ chromosome micro deletion analysis are recommended in patients with azoospermia and sperm concentrations less than 10 million per $\mathrm{ml}$ before planning assisted reproduction treatment. $\mathrm{Y}$ chromosome (Yq) micro deletions are associated with male infertility. Genetic counseling should be carried out in such patients, as the $\mathrm{Y}$ chromosome deletions will be passed on to the male child produced through assisted reproduction techniques.

In obstructive azoospermia, spermatogenesis is usually fully preserved as it results due to physical disruption of the seminal tract, the cause for which may be either congenital or caused by trauma (surgical or non-surgical) or infection. Surgical reconstruction in form of vasovasostomy or vasoepididimostomy can be considered a cost effective method for treatment of obstructive azoospermia. $^{14,15}$ In such cases, at the time of reconstruction, the testicular biopsy can be done and sperm cryopreserved as well for future use in case of procedural failure. But patients with $\mathrm{OA}$ who are not candidates for reconstructive surgery and those who fail to achieve success with such procedures and patients with NOA can be treated by surgical sperm retrieval and ICSI.

Surgical Sperm retrieval techniques (MESA, PESA, TESA, TESE and TEFNA) have been developed to obtain spermatozoa from the epididymis and testicles of azoospermic men seeking fertility treatment. Either simple washing or gradient centrifugation is done to obtain sperms for ICSI processes post surgical retrieval procedure. ${ }^{16}$ The use of non-ejaculated sperm and intracytoplasmic sperm injection (ICSI) has become an established procedure for patients suffering from azoospermia.

\section{METHODS}

Men recruited in the study underwent thorough history taking, examination, hormonal assay, and genetic analysis before surgical sperm retrieval. In all cases, sperm retrieval was first attempted by per cutaneous epididymal sperm aspiration (PESA), and if unsuccessful then the patient was taken up for testicular sperm extraction (TESE). The protocol (long or antagonist) used for controlled ovarian stimulation varied on the basis of physician preference, patient and clinical scenario. As 
part of this study center's routine protocol, embryo transfer was done on D-2/3 i.e. at 6-8-cell stage. The serum quantitative $\beta \mathrm{HCG}$ concentrations were taken on day 16 after embryo transfer. Initial ultrasound was done 18 days post embryo transfer to verify intrauterine pregnancy and numbers of gestational sacs followed by a repeat scan after one week.

\section{Statistical analysis}

The descriptive statistics was done by using mean or median with standard deviation (SD) or inter quartile range (IQR) for quantitative variables and categorical variables were presented in frequencies along with respective percentages. The best cut-off for FSH, LH and Testosterone was computed by sensitivity, specificity, Positive Predictive Value (PPV), Negative Predictive Value (NPV) and Receiver Operative Characteristic (ROC). The other statistical comparison for quantitative values for two groups was done by Mann-Whitney ' $U$ ' test and that for three groups was done by KrushkaWallies "H" test. The categorical variables were compared by Chi-square test. All statistical analyses were performed using SPSS software (Version 22, SPSS Inc, Chicago, IL, USA). The $\mathrm{p}$ value less than 0.05 were considered as statistically significant.

\section{RESULTS}

A total of 100 patients known case of azoospermia underwent surgical sperm retrieval followed by ICSI whereas 100 patients underwent ICSI with ejaculated sperms for indications like oligoasthenospermia or fertilization failure in the previous cycle between Jan 2016 and Dec 2016. Patients who had concomitant female factor like endometriosis, fibroids, etc. that could affect outcome were not included in the study. Patient's characteristic and number of oocytes retrieved, number of M-II oocytes and number of Gd 1 embryos obtained are shown in Table 1.

Table 1: Patients characteristics, number of oocytes and embryos obtained in the study.

\begin{tabular}{|lll|}
\hline & $\begin{array}{l}\text { Surgical sperm } \\
\text { retrieval+ICSI } \\
\text { group }\end{array}$ & $\begin{array}{l}\text { Only ICSI } \\
\text { group }\end{array}$ \\
\hline Male patient age (y) & $32.76 \pm 3.420$ & $31.27 \pm 5.07$ \\
\hline Female patient age (y) & $29.91 \pm 3.817$ & $27.06 \pm 3.641$ \\
\hline $\begin{array}{l}\text { No. of oocytes } \\
\text { retrieved }\end{array}$ & $8.73 \pm 4.786$ & $8.97 \pm 5.576$ \\
\hline No. of MII oocytes & $5.65 \pm 3.192$ & $5.78 \pm 3.393$ \\
\hline No. of Gd 1 embryos & $3.57 \pm 2.170$ & $3.23 \pm 1.823$ \\
\hline Fertilization failure & $5.2 \%$ & $4 \%$ \\
\hline Implantation rate & $53.94 \%$ & $53 \%$ \\
\hline
\end{tabular}

The mean male age in surgical retrieval group was $32.76 \pm 3.420$ versus 31.27 years \pm 5.07 for ICSI group and the mean female age in surgical retrieval group was $29.91 \pm 3.817$ versus 27.06 years \pm 3.641 for ICSI group, showing that both male and female partners were older in surgical retrieval group but was not statistically significant (Table 1).

100 azoospermic men first underwent PESA. Out of 100 patients in $47,(47.0 \%)$ patient's sperms were retrieved by PESA. The remaining 53 patients in whom PESA could not extract sperms, TESE were attempted in the same sitting. Among 53 patients who underwent TESE sperms were retrieved in $29(54.7 \%)$ patients. The success rate of surgical sperm retrieval (PESA + TESE) in our study was $76.0 \%$.

Table 2: Outcome of PESA in the study.

\begin{tabular}{|lllllll|}
\hline Outcome & Negative & Positive & FF & Oocyte freezing & \multicolumn{2}{c|}{ Nil oocyte retrieval } \\
\hline $\begin{array}{l}\text { PESA } \\
\text { (Positive) }\end{array}$ & 19 & 25 & 1 & 1 & 1 & 47 \\
\hline
\end{tabular}

Table 3: Outcome of TESE in the study.

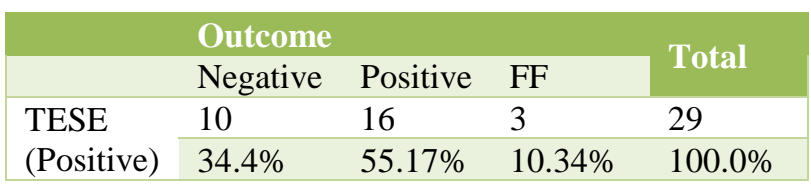

Among 47 PESA positive patients one patient had nil oocyte retrieval and hence was not considered for result outcome analysis. Implantation rate was $53.19 \%, 55.17 \%$ and $53.0 \%$ respectively (Table 2,3 and 4 ) in PESA,
TESE and ICSI with ejaculated sperm groups. Cumulative implantation rate of PESA and TESE was

Table 4: Outcome of ICSI in the study.

\begin{tabular}{|lllll|}
\hline & Outcome & & & \\
& Negative & Positive & FF & Total \\
\hline \multirow{2}{*}{ ICSI } & 43 & 53 & 4 & 100 \\
& $(43.0 \%)$ & $(53.0 \%)$ & $(4.0 \%)$ & $(100 \%)$ \\
\hline
\end{tabular}

$53.94 \%$ (Table 5). There were $3(10.34 \%)$ fertilization failure in TESE group, $1(2.1 \%)$ in PESA group and 4 
$(4.0 \%)$ in only ICSI group. There was no statistical difference concerning fertilization rate, embryo quality and pregnancy rate between ICSI with surgically retrieved sperm and ejaculated sperms.

Table 5: Outcome of PESA and TESE in the study.

\begin{tabular}{|lllllll|}
\hline & $\begin{array}{l}\text { Outcome } \\
\text { Negative }\end{array}$ & Positive & FF & Oocyte freezing & Nil oocyte retrieval & \multicolumn{2}{c|}{ Total } \\
\hline PESA and TESE & 29 & 41 & 4 & 1 & 1 & 76 \\
(Positive) & $38.15 \%$ & $53.94 \%$ & $5.2 \%$ & $1.31 \%$ & $1.31 \%$ & $100.0 \%$ \\
\hline
\end{tabular}

The cut-off value of FSH for successful surgical sperm retrieval was $8.27 \mathrm{mIU} / \mathrm{mL}$ with sensitivity and specificity of $76.9 \%$ and $75.5 \%$ respectively (Figure 1).

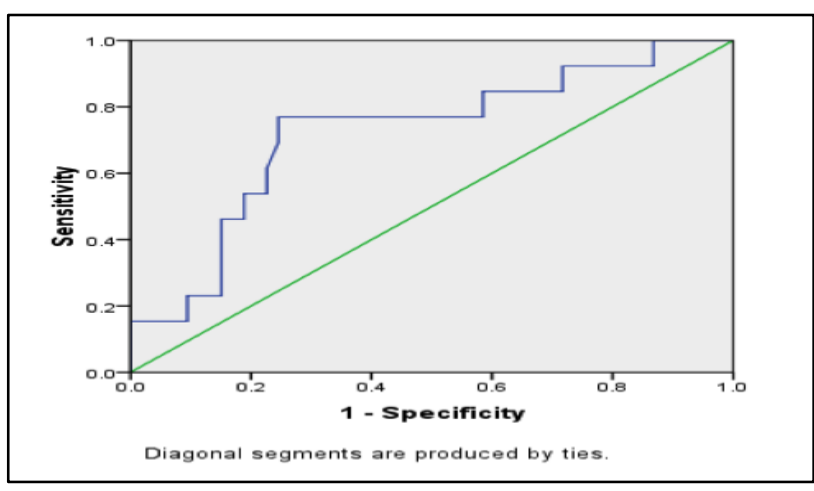

Figure 1: ROC Curve: FSH.

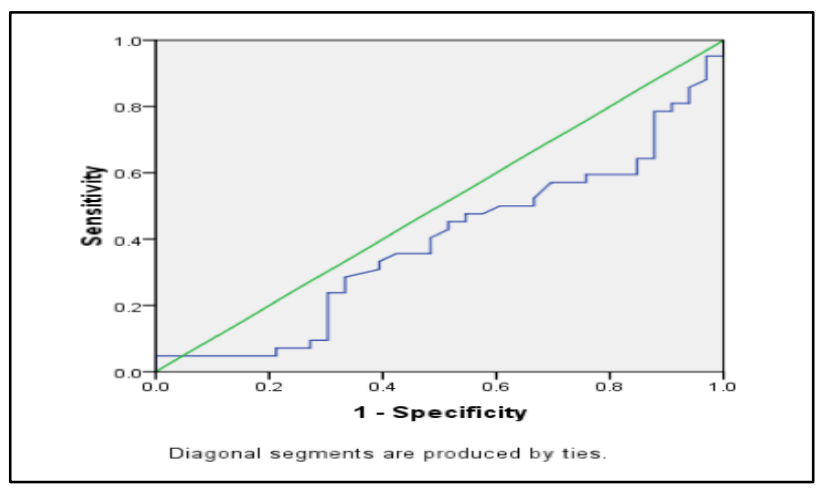

Figure 2: ROC Curve: LH.

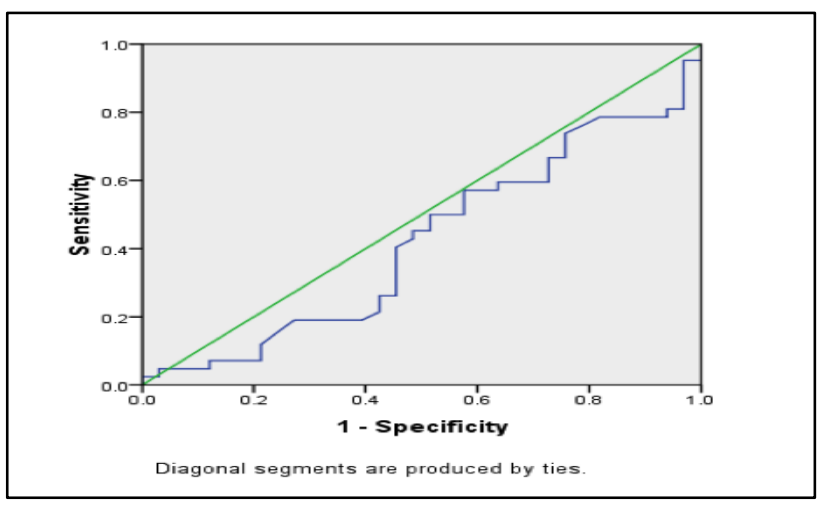

Figure 3: ROC Curve: Testosterone.
LH and Testosterone levels were not very sensitive and specific parameters for predicting outcome of surgical sperm retrieval (Figure 2 and 3 ).

In our series of 100 patients who underwent surgical retrieval one patient developed mild inflammation post PESA and was managed with antibiotics as an outpatient, and one patient had hematoma post TESE, which needed hospitalization.

\section{DISCUSSION}

Infertility is mainly associated with the female is a commonly faced misconception in the management of infertility especially in developing countries like ours. Due to a comprehensive evaluation of male reproductive function and improved diagnostic tools, the role of the male factor in infertility has been exponentially rising in recent years. The treatment of male factor infertility has been more empirical rather than evidence based. Assisted Reproductive Techniques has proved a better adjunctive treatment for alleviating male factor infertility where empirical treatments are not indicated or have failed.

Despite the improvements in medical science, azoospermia is always the most challenging topic associated with infertility treatment. The options when treating patients with azoospermia are the choice between using surgical correction of obstruction, surgically retrieved sperm or resorting to using of donor sperms. Patients are reluctant to use donor sperm, as it does not give the patient their genetically own children.

Over the past two decades treatment of azoospermia has seen radical change. Surgical sperm retrieval (SSR) and ICSI has made azoospermia a diagnosis, which is amenable to treatments. SSR has helped more than $95 \%$ males to father their own genetic child.

In patients with azoospermia where surgical correction is not feasible or has failed, recovery of spermatozoa can be made from either the epididymis or the testis. Surgical sperm retrieval and ICSI provided the opportunity for men with azoospermia to father biological children. ICSI, is performed when a single selected sperm is injected into a denuded egg instead of simply placing millions of sperm in a solution with the egg as performed in traditional IVF. Hence only a few sperm are sufficient for 
fertilization with ICSI, which enabled techniques of surgical sperm retrieval to be performed in the management of azoospermia. Different surgical techniques such as percutaneous epididymal sperm aspiration (PESA), testicular sperm extraction (TESE), microsurgical epididymal sperm aspiration (MESA), testicular sperm aspiration (TESA) and fine-needle aspiration of the testis have been described for sperm retrieval. Surgically retrieval can be done on the day of ovum pick up and retrieved sperm can be used in the same cycle or can be done prior and sperms can be cryopreserved for future use. A Cochrane meta-analysis on surgical sperm retrieval techniques has also been unable to recommend any specific sperm retrieval technique. ${ }^{17}$ Technique of choice depends on etiology of azoospermia and surgeon's preference and expertise. ${ }^{18}$

The three main goals that any technique of Sperm retrieval used should address are:

- To be able to obtain an adequate number of sperm for both immediate use and cryopreservation for future use so patient is not subjected to surgical procedure again and again;

- To retrieve best quality sperms;

- To minimize damage to the reproductive tract so that future sperm retrieval attempts (if required) or testicular function are not jeopardized. ${ }^{19}$

In cases of azoospermia especially obstructive type epididymal sperm aspiration is the first choice for surgical sperm retrieval but if PESA is negative or in cases of non-obstructive azoospermia testicular sperm retrieval is an accepted alternative. However, chances of complications such as inflammation and hematoma are more likely with testicular sperm retrieval.

Testicular sperm extraction technique was first reported in 1993. The first pregnancy with use of frozen epididymal sperm and testicular sperm was achieved in 1995 and 1996 respectively. ${ }^{20}$

Sperm retrieval rate in our study was $76 \%$ when PESA and TESE are combined though studies have reported $70 \%$ to $100 \%$ retrieval when percutaneous epididymal and testicular retrievals are combined. ${ }^{21}$

In our series, TESE was done in $33 \%$ patients as a rescue procedure after failed PESA whereas, Esteves et al. had to revert to rescue procedure in only $17 \%$ cases. $^{22}$

No difference in fertilization rates was found between the ICSI attempts performed with ejaculated and surgically retrieved spermatozoa in infertile men. There was also no difference in pregnancy outcome between two groups, which is in consensus with many studies. ${ }^{22,23}$ However, some studies have found lower outcome of ICSI with surgically retrieved sperm vis ejaculated sperms. ${ }^{24}$
In our patients, results of ICSI cycles using epididymal and testicular sperm had similar outcomes showing that source of sperm or etiology does not affect sperm injection outcome as also shown in other studies. ${ }^{25}$

Our data confirm a similar outcome of ICSI in men with azoospermia using either epididymal or testicular sperm, with patients who underwent ICSI with ejaculated sample. ${ }^{26}$ In literature, no threshold value has been found for FSH, inhibin B, or testicular volume to predict successful sperm harvesting, cut-off value of FSH for successful surgical sperm retrieval in our study was 8.27 $\mathrm{mIU} / \mathrm{mL}$ with sensitivity and specificity of $76.9 \%$ and $75.5 \%$ respectively. ${ }^{27}$

Some studies have shown that use of microsurgery for TESE may further increase retrieval rates as there may be focal spermatogenesis in men with non obstructive azoospermia. $^{28}$ Human spermatozoa are highly specialized cells with the purpose of delivering competent paternal DNA to the oocyte and providing a robust epigenetic contribution to embryogenesis. ${ }^{29}$

\section{CONCLUSION}

Absence of sperms in the ejaculate should not label a man sterile. Attempts should be made to overcome obstacles in path of an azoospermic male in fathering own child. All males with azoospermia irrespective of their history and evaluation findings should be given a chance to father genetically own child by attempting surgical sperm retrieval. A coordinated multidisciplinary effort is key to offer the best possible chance of achieving a biological offspring for males with azoospermia.

Funding: No funding sources Conflict of interest: None declared

Ethical approval: The study was approved by the Institutional Ethics Committee

\section{REFERENCES}

1. World Health Organization. WHO Laboratory Manual for the Examination and Processing of Human Semen. $5^{\text {th }}$ ed. 2010. Available at http://apps.who.int/iris/bitstream/10665/44261/1/978 9241547789_eng.pdf

2. Aziz N. The importance of semen analysis in the context of azoospermia. Clinics (Sao Paulo). 2013;68(Suppl 1):35-8.

3. Esteves SC. Clinical relevance of routine semen analysis and controversies surrounding the 2010 World Health Organization criteria for semen examination. Int Braz J Urol. 2014;40:443-53.

4. Esteves SC, Miyaoka R, Agarwal A. An update on the clinical assessment of the infertile male. Clinics. 2011;66(4):691-700.

5. Samplaski MK, Lo K, Grober E, Jarvi K. Finasteride use in the male infertility population: Effects on 
semen and hormone parameters. Fertil Steril. 2013;100:1542-6.

6. Baker K, Sabanegh E. Obstructive azoospermia: reconstructive techniques and results. Clinics (Sao Paulo). 2013;68(Suppl 1):61-73.

7. Esteves SC, Lee W, Benjamin DJ, Seol B, Verza A, Jr. Reproductive potential including neonatal outcomes of men with obstructive azoospermia undergoing percutaneous sperm retrieval and intracytoplasmic sperm injection according to the cause of obstruction. J Urol. 2013;189:232-7.

8. Miyaoka R, Esteves SC. Predictive factors for sperm retrieval and sperm injection outcomes in obstructive azoospermia: do etiology, retrieval techniques and gamete source play a role? Clinics (Sao Paulo). 2013;68(Suppl 1):111-9.

9. Esteves SC, Agarwai A. The azoospermic male: current knowledge and future perspectives. Clinics. (Sao Paulo) 2013;68(Suppl 1):1-4.

10. Hung AJ, King P, Schlegel PN. Uniform testicular maturation arrest: a unique subset of men with nonobstructive azoospermia. J Urol. 2007;178:60812.

11. Sokol RZ, Swerdloff RS. Endocrine evaluation. In: Lipshultz LI, Howards SS, editors. Infertility in the Male. 3rd ed. New York: Churchill Livingstone; 1997:210-8.

12. Gudeloglu A, Parekattil SJ. Update in the evaluation of the azoospermic male. Clinics (Sao Paulo). 2013;68(Suppl 1):27-34.

13. Esteves SC, Hamada A, Kondray V, Pitchika A, Agarwal A. What every gynecologist should know about male infertility: an update. Arch Gynecol Obstet. 2012;286:217-29.

14. Lee R, Li PS, Goldstein M, Tanrikut C, Schattman G, Schlegel PN. A decision analysis of treatments for obstructive azoospermia. Hum Reprod. 2008;23(9):2043-9.

15. Lee R, Li PS, Schlegel PN, Goldstein M. Reassessing reconstruction in the management of obstructive azoospermia: reconstruction or sperm acquisition? Urol Clin North Am. 2008; 35(2): 289301.

16. Ozkavukcu S, Ibis E, Kizil S, Isbacar S, Aydos K. A laboratory modification to testicular sperm preparation technique improves spermatogenic cell yield. Asian J Androl. 2014;16:852-7.

17. van Peperstraten A, Proctor ML, Johnson NP, Philipson G. Techniques for surgical retrieval of sperm prior to intra-cytoplasmic sperm injection (ICSI) for azoospermia. Cochrane Database Syst Rev. 2008.

18. Esteves SC, Miyaoka R, Orosz JE, Agarwal A. An update on sperm retrieval techniques for azoospermic males. Clinics [Sao Paulo]. 2013:68 Suppl 1:99-110
19. Esteves SC, Miyaoka R, Agarwal A. Sperm retrieval techniques for assisted reproduction. Int Braz J Urol. 2011;37:570-83.

20. Verheyen Greta Outcomes, safety and effectiveness for cryopreservation of ejaculated, testicular and epididymal sperm. Centre for Reproductive Medicine UZ Brussel, Belgium. ALPHA Conference Budapest 30th April - 2nd. May 2010. Available at http://www.brusselsivf.be/dissertations.

21. Naredi N, Agrawal. A Testicular sperm extraction: can we combat both obstructive and non obstructive azoospermia?. Endocrinol Metab Syndr. 2016;5:218.

22. Esteves SC, Lee W, Benjamin DJ, Seol B, Verza S Jr, Agarwal A. Reproductive potential of men with obstructive azoospermia undergoing percutaneous sperm retrieval and intracytoplasmic sperm injection according to the cause of obstruction. $\mathrm{J}$ Urol. 2013;189(1):232-7.

23. Fahmy I, Mansour R, Aboulghar M, Serour G, Kamal A, Tawab NA et al. Intracytoplasmic sperm injection using surgically retrieved epididymal and testicular spermatozoa in cases of obstructive and non-obstructive azoospermia. Int $\mathrm{J}$ Androl. 1997;20:37-44.

24. Palermo GD, Sclegel PN, Hariprashad JJ, Ergun B, Mienik A, Zaninovic $\mathrm{N}$ et al. Fertilisation and pregnancy outcome with intracytoplasmic sperm injection for azoospermic men. Hum Reprod. 1999; 14:741-8.

25. Nicopoullos JDM, Gilling-Smith C, Almeida PA, Ramsay JWA. The results of 154 ICSI cycles using surgically retrieved sperm from azoospermic men. HumReprod. 2004;19(3):579-85.

26. Kamal A, Fahmy I, Mansour R, Serour G, Aboulghar M, Ramos L, et al. Does the outcome of ICSI in cases of obstructive azoospermia depend on the origin of the retrieved spermatozoa or the cause of obstruction? A comparative analysis. Fertil Steril. 2010;94 (6):2135-40.

27. Abdel-Meguid TA. Predictors of sperm recovery and azoospermia relapse in men with nonobstructive azoospermia after varicocele repair. J Urol. 2012;187(1):222-6.

28. Ran R, Kohn TP, Ramasamy R. Innovation in surgical management of non obstructive azoospermia. Indian J Urol. 2016;32(1):15-20.

29. Kumar M, Kumar K, Jain S, Hassan T, Dada R. Novel insights into the genetic and epigenetic paternal contribution to the human embryo. Clinics (Sao Paulo). 2013;68(Suppl 1):5-14.

Cite this article as: Rai S, Rao PS, Prajwal S. Sperm recovery and IVF outcome after surgical sperm retrieval in azoospermia: our experience. Int $\mathrm{J}$ Reprod Contracept Obstet Gynecol 2018;7:xxx-xx. 